\title{
The impact of BMI on sperm parameters and the metabolite changes of seminal plasma concomitantly
}

\author{
Dan Guo ${ }^{1,2, *}$, Wei Wu ${ }^{1,2,3, *}$, Qiuqin Tang ${ }^{4, *}$, Shanlei Qiao ${ }^{1,2}$, Yiqiu Chen ${ }^{1,2}$, Minjian \\ Chen $^{1,2}$, Mengying Teng ${ }^{1,2}$, Chuncheng Lu ${ }^{1,2}$, Hongjuan Ding ${ }^{4}$, Yankai Xia ${ }^{1,2}$, Lingqing \\ $\mathrm{Hu}^{3}$, Daozhen Chen ${ }^{3}$, Jiahao Sha ${ }^{5}$ and Xinru Wang ${ }^{1,2}$ \\ ${ }^{1}$ State Key Laboratory of Reproductive Medicine, Institute of Toxicology, School of Public Health, Nanjing Medical University, \\ Nanjing, China \\ ${ }^{2}$ Key Laboratory of Modern Toxicology of Ministry of Education, Nanjing Medical University, Nanjing, China \\ ${ }^{3}$ State Key Laboratory of Reproductive Medicine, Wuxi Maternal and Child Health Care Hospital Affiliated to Nanjing Medical \\ University, Wuxi, China \\ ${ }^{4}$ Department of Obstetrics, State Key Laboratory of Reproductive Medicine, Obstetrics and Gynecology Hospital Affiliated to \\ Nanjing Medical University, Nanjing, China \\ ${ }^{5}$ State Key Laboratory of Reproductive Medicine, Nanjing Medical University, Nanjing, China \\ * These authors have contributed equally to this study \\ Correspondence to: Wei Wu, email: wwu@njmu.edu.cn
}

Xinru Wang, email: xrwang@njmu.edu.cn

Keywords: BMl, obesity, sperm parameters, meta-analysis, metabolomic analysis, Pathology Section

Received: April 26, $2016 \quad$ Accepted: December 16, $2016 \quad$ Published: February 01, 2017

Copyright: Guo et al. This is an open-access article distributed under the terms of the Creative Commons Attribution License 3.0 (CC BY 3.0), which permits unrestricted use, distribution, and reproduction in any medium, provided the original author and source are credited.

\section{ABSTRACT}

The development of male infertility increased rapidly worldwide, which coinciding with the epidemic of obesity. However, the impact of weight abnormalities on sperm quality is still contestable. To assess the correlation between BMI and sperm parameters, we searched relevant articles in PubMed, Embase, Web of science, and Wanfang database published until June $\mathbf{2 0 1 5}$ without language restriction. Otherwise, we also recruited some participants who attended fertility clinic as well as some general populations in this report. We performed a systematic review and meta-analysis about BMI and sperm parameters containing total sperm count, concentration, semen volume and sperm motility (overall and progressive). Metabolomic analysis of seminal plasma was performed to explore the mechanism from a new perspective. This study found standardized weighted mean differences (SMD) in sperm parameters (total sperm count, sperm concentration, and semen volume) of abnormal weight groups decreased to different degree compared to normal weight. Dose-response analysis found SMD of sperm count, sperm concentration and semen volume respectively fell $2.4 \%, 1.3 \%$ and $2.0 \%$ compared with normal weight for every 5 -unit increase in BMI. Metabolomic analysis of seminal plasma showed that spermidine and spermine were likely to play a vital role in the spermatogenesis progress. This systematic review with meta-analysis has confirmed there was a relationship between BMI and sperm quality, suggesting obesity may be a detrimental factor of male infertility.

\section{INTRODUCTION}

The development of assisted reproductive technology and its exponential increase usage have reflected, to a certain extent, infertility has become a serious worldwide problem. Forty-eight point five million couples were infertile in 2010, and about 50\% infertility is caused by male factors $[1,2]$. Sperm quality and spermatogenesis is vital for male fertility. The stand or fall of these situations depends on multi-factors i.e. genetic, environmental, behavioral or dietary. As a growing social health problem, the effect of obesity is not to be sneezed at 
that costs to both the community and the individual since obesity may be related to cardiovascular disease, diabetes and cancers $[3,4]$. Along with the prevalence of obesity in the world that the proportion of men who are overweight (BMI $\geqq 25$ ) is $36.9 \%$ in 2013 [5], and at the same time, infertility often coexists with obesity, many investigators put their eyes on the impact of body mass index on sperm quality. Since the beginning of this century, relevant researches emerged in endlessly. However, there is still a debate about whether overweight/obesity is a risk factor for infertility. Shayeb et al (2011) and Duits et al (2010) found high BMI mainly caused low semen volume and had no effect on other sperm parameters $[6,7]$. Stephanie Belloc et al concluded increased BMI affected sperm quality including sperm count, concentration, volume and motility [8]. However, Aggerholm et al (2008) got the conclusion that there was no statistically significant relationship between BMI and sperm count and concentration [9]. Two meta-analyses published in 2010 and 2013 revealed different conclusions that MacDonald et al found no relationship between obesity and sperm concentration or sperm count, while Sermondade et al showed obesity increased the risk of abnormal sperm count $[10,11]$. Since 2013, many new relevant researches published in succession, including some consistent with the conclusion of MacDonald et al [12-15], and some got the same result with Sermondade et al [8, 16-19]. It was surprising the participant numbers of some studies are unprecedented, for instance, Stephanie Belloc et al recruited 10665 men and Chih-Wei Tsao et al recruited 7630 men to evaluate the association between BMI and semen characteristics in 2014 and 2015, respectively [8, $18]$.

We summarized these new studies combined with the past as well as our personal data, and conducted this study to investigate the effect of overweight and obesity on several sperm parameters. Besides, because obesity is a metabolic disorder phenomenon, a proper understanding of small molecule metabolites in human seminal plasma will provide biological information on mechanism underlying spermatogenesis. Therefore, we also performed the metabolomic analysis of seminal plasma in this study.

\section{RESULTS}

\section{Result of search}

The search strategy identified a total of 31524 articles, including 9382 from PubMed, 11515 from Web of Science, 9277 from Embase and 1350 from Wanfang; however, 31089 studies were excluded because 14242 were reduplicative and 16847 articles had no relevance to the primary research. We selected 42 articles that providing BMI and sperm parameters data after review

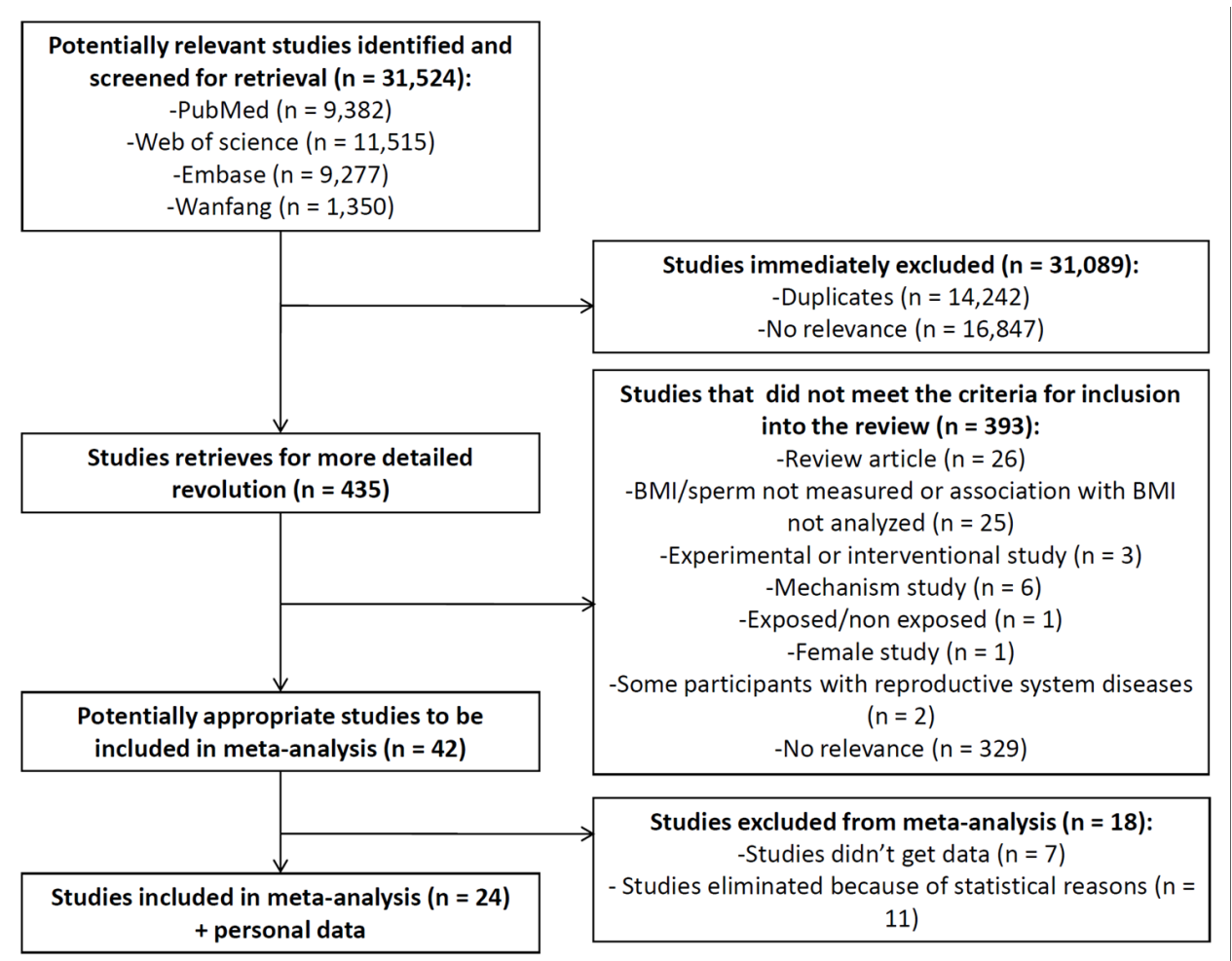

Figure 1: Flow chart of study selection. 
Table 1: Characteristics of observational studies included in this review (Part 1)

\begin{tabular}{|c|c|c|c|c|c|c|c|c|c|c|}
\hline \multirow{2}{*}{ Study } & \multirow{2}{*}{ Location } & \multirow{2}{*}{ Study group } & \multirow{2}{*}{$\begin{array}{l}\text { Ascertainment } \\
\text { of BMI }\end{array}$} & \multirow{2}{*}{$\begin{array}{l}\text { Repeated } \\
\text { semen } \\
\text { collection }\end{array}$} & \multirow{2}{*}{ BMI distribution } & \multirow{2}{*}{$\begin{array}{l}\text { Total sperm count } \\
\begin{array}{l}\text { Mean } \pm \text { SD/ Median } \\
(\mathrm{IQR})\end{array}\end{array}$} & $\begin{array}{l}\text { Sperm } \\
\text { concentration }\end{array}$ & Semen volume & Sperm motility & $\begin{array}{l}\text { Sperm motility } \\
\text { (progressive) }\end{array}$ \\
\hline & & & & & & & $\begin{array}{l}\text { Mean } \underset{\text { Median }(\mathrm{IQR})}{ \pm} \mathrm{SD} / \\
\text { Medila }\end{array}$ & $\begin{array}{l}\text { Mean } \pm \text { SD/ } \\
\text { Median }(\mathrm{IQR})\end{array}$ & $\begin{array}{l}\text { Mean } \pm \text { SD/ } \\
\text { Median (IQR) }\end{array}$ & $\begin{array}{l}\text { Mean } \pm \text { SD/ } \\
\text { Median }(I Q R)\end{array}$ \\
\hline \multirow{5}{*}{$\begin{array}{l}\text { Belloc et al } \\
\quad(2014)\end{array}$} & \multirow{5}{*}{ France } & \multirow{5}{*}{$\begin{array}{l}10170 \text { men referred in the } \\
\text { course of a couple infertility } \\
\text { evaluation of any origin }\end{array}$} & \multirow{5}{*}{ Self-reported } & \multirow{5}{*}{ once } & $18.5-24.9(\mathrm{n}=5799)$ & $171 \pm 170$ & $56.4 \pm 54.9$ & $3.3 \pm 1.6$ & $39.7 \pm 16.7$ & $36.9 \pm 16.8$ \\
\hline & & & & & $25.0-29.9(\mathrm{n}=3607)$ & $163 \pm 175$ & $55.1 \pm 56.9$ & $3.2 \pm 1.5$ & $39.4 \pm 16.6$ & $36.5 \pm 16.8$ \\
\hline & & & & & $30.0-34.9(n=634)$ & $141 \pm 166$ & $50.7 \pm 55.7$ & $3.1 \pm 1.6$ & $37.5 \pm 16.6$ & $34.4 \pm 16.9$ \\
\hline & & & & & $35.0-39.9(\mathrm{n}=97)$ & $136 \pm 144$ & $49.7 \pm 49.5$ & $3.0 \pm 1.8$ & $38.5 \pm 15.8$ & $35.6 \pm 15.9$ \\
\hline & & & & & $\geq 40.0(\mathrm{n}=33)$ & $92 \pm 95$ & $39.4 \pm 51.0$ & $2.7 \pm 1.6$ & $38.0 \pm 16.2$ & $34.7 \pm 17.1$ \\
\hline \multirow{3}{*}{$\begin{array}{c}\text { Guo et al } \\
\text { (unpublished) }\end{array}$} & \multirow{3}{*}{ China } & \multirow{3}{*}{$\begin{array}{l}2106 \text { men including } 645 \text { fertile } \\
\text { men and } 1461 \text { infertile men } \\
\text { from maternity clinic and } \\
\text { infertility clinic respectively }\end{array}$} & \multirow{3}{*}{ Self-reported } & \multirow{3}{*}{ once } & $18.5-24.0(\mathrm{n}=1057)$ & $157.94 \pm 201.33$ & $49.90 \pm 43.60$ & $3.18 \pm 2.32$ & $46.98 \pm 23.86$ & $37.76 \pm 20.59$ \\
\hline & & & & & $24.1-27.9(\mathrm{n}=798)$ & $154.32 \pm 165.89$ & $50.90 \pm 45.73$ & $3.07 \pm 1.52$ & $47.70 \pm 23.37$ & $39.06 \pm 20.33$ \\
\hline & & & & & $\geq 28.0(\mathrm{n}=251)$ & $141.26 \pm 143.80$ & $49.06 \pm 39.53$ & $2.91 \pm 1.43$ & $47.97 \pm 23.83$ & $39.14 \pm 21.22$ \\
\hline \multirow{3}{*}{$\begin{array}{l}\text { Paasch et al } \\
\quad(2010)\end{array}$} & \multirow{3}{*}{ Germany } & & & & $20-25(\mathrm{n}=1003)$ & $159.2 \pm 5.51 \mathrm{a}$ & & & $40.9 \pm 1.01^{\mathrm{a}}$ & \\
\hline & & the factors affecting semen & NA & once & $25-30(\mathrm{n}=810)$ & $143.7 \pm 6.26 \mathrm{a}$ & & & $39.7 \pm 0.99^{\mathrm{a}}$ & \\
\hline & & $\begin{array}{l}\text { related diseases and chronic } \\
\text { diseases }\end{array}$ & & & $\geq 30(\mathrm{n}=245)$ & $143.0 \pm 11.68 \mathrm{a}$ & & & $38.1 \pm 2.06^{\mathrm{a}}$ & \\
\hline & & 2017 male partners of couples & & & 18.5-24.99 $(\mathrm{n}=839)$ & $144.0(61.1-290.8)^{c}$ & $47.9(22.0-84.3)^{c}$ & $3.5 \pm 1.8$ & $45.0(29.4-59.0)^{\mathrm{c}}$ & \\
\hline $\begin{array}{l}\text { Shayeb et al } \\
\text { (2011) }\end{array}$ & $\begin{array}{l}\text { United } \\
\text { Kingdom }\end{array}$ & investigations at the Aberdeen & NA & once & $25.0-29.99(\mathrm{n}=909)$ & $153.0(58.8-273.4)^{\mathrm{c}}$ & $47.0(21.0-82.0)^{c}$ & $3.5 \pm 1.8$ & $45.4(27.6-59.0)^{\circ}$ & \\
\hline & & 2007 & & & $\geq 30(\mathrm{n}=269)$ & $162.7(48.9-259.8)^{\mathrm{c}}$ & $50.8(21.3-83.0)^{\mathrm{c}}$ & $3.2 \pm 1.7$ & $47.0(27.0-61.0)^{\mathrm{c}}$ & \\
\hline & & 1922 men without prior & & & $20.0-25.0(\mathrm{n}=986)$ & $161(77-309)$ & $55(9-99)$ & & & $40(19-66)$ \\
\hline $\begin{array}{l}\text { Aggerholm et al } \\
(2008)\end{array}$ & Denmark & aged 18-66 from five separate & Self-reported & once & $25.1-30.0(\mathrm{n}=773)$ & $153(67-286)$ & $53(27-90)$ & & & $52(28-66)$ \\
\hline & & semen studies & & & $>30.0(\mathrm{n}=163)$ & $156(75-317)$ & $65(33-114)$ & & & $59(40-72)$ \\
\hline & & 1341 all 18-year-old men & & & $20-25(\mathrm{n}=1042)$ & $138.0(59-259)$ & $46.0(23-84)$ & $3.2 \pm 1.4$ & $65.4 \pm 12.4$ & \\
\hline$(2004)$ & Denmark & $\begin{array}{l}\text { examination for military service } \\
\text { excluding chronic diseases }\end{array}$ & Measured on site & once & $\geq 25(\mathrm{n}=299)$ & $116.0(46-213)$ & $39.0(20-69)$ & $3.2 \pm 1.6$ & $65.5 \pm 12.5$ & \\
\hline & & 1366 men visiting the Centre & & & $20.1-25(\mathrm{n}=633)$ & $174.3(50.2-219.6)$ & $53.3(18.0-89.0)$ & $3.7 \pm 2.5$ & & $31.1 \pm 15.9$ \\
\hline $\begin{array}{l}\text { Duits et al } \\
(2010)\end{array}$ & $\begin{array}{l}\text { the } \\
\text { Netherlands }\end{array}$ & $\begin{array}{l}\text { for Reproductive Medicine as } \\
\text { part of a subfertile couple from }\end{array}$ & Self-reported & two semen & $25.1-30(\mathrm{n}=587)$ & $153(48.8-283.7)$ & $56(17.8-96.8)$ & $3.3 \pm 1.7$ & & $32.5 \pm 15.9$ \\
\hline & & 2000 to 2007 & & & $>30(\mathrm{n}=146)$ & $135.6(45.8-261.6)$ & $47(17.4-86.5)$ & $3.4 \pm 1.6$ & & $33.5 \pm 16.2$ \\
\hline & & & & & $18.5-23.9(n=401)$ & $166.5(70.4-298)$ & $71(27-111)$ & $2.5(2-3.5)$ & & $48(28.0-60.0)$ \\
\hline Xiao et al & Ching & 786 men attending the & Mensured on site & once & $24-27.9(\mathrm{n}=221)$ & $126(64.6-215)$ & $70(32-98.5)$ & $2(1.5-3)$ & & $48.0(31.5-59.5)$ \\
\hline (2013) & minia & clinic in 2010-2013 & Mivedsured on sile & once & $28-29.9(\mathrm{n}=120)$ & $121(62.3-215.1)$ & $63(28-103)$ & $2(1.3-2.8)$ & & $45(35.0-57.0)$ \\
\hline & & & & & $\geq 30(n=44)$ & $125(46.1-288.8)$ & $64.5(37.3-112.3)$ & $2(1.3-2.5)$ & & $44.5(33.3-54.5)$ \\
\hline & & 794 male partner of couples & & & $18.5-24.9(\mathrm{n}=251)$ & & $43.7 \pm 1.9 \mathrm{a}$ & $3.2 \pm 0.1 \mathrm{a}$ & $51.4 \pm 1.2^{\mathrm{a}}$ & $39.8 \pm 1.2^{\mathrm{a}}$ \\
\hline $\begin{array}{l}\text { Martini et al } \\
(2010)\end{array}$ & Argentina & $\begin{array}{l}\text { attending the Andrology and } \\
\text { Reproduction Laboratory in }\end{array}$ & Measured on site & once & $25.0-29.9(\mathrm{n}=388)$ & & $44.2 \pm 1.8 \mathrm{a}$ & $3.1 \pm 0.1 \mathrm{a}$ & $50.2 \pm 1.0^{\mathrm{a}}$ & $38.8 \pm 0.9^{a}$ \\
\hline & & 2007 , Argentina in $2000-$ & & & $30-50(\mathrm{n}=155)$ & & $43 \pm 3.2 \mathrm{a}$ & $3.1 \pm 0.1 \mathrm{a}$ & $46.6 \pm 1.7^{\mathrm{a}}$ & $35.9 \pm 1.6^{\mathrm{a}}$ \\
\hline & & & & & $<25(\mathrm{n}=83)$ & $198.5(112.8-336.9)$ & $55.3(34.4-94.1)$ & $3.5(2.4-4.8)$ & $70.7(64.8-75.5)$ & \\
\hline Eisenberg et al & USA & to conceive in two geographic & Measured on site & once or twice & $25-30(n=191)$ & $190.6(100.3-338.1)$ & $63.2(38.0-92.5)$ & $3.4(2.1-4.7)$ & $67.6(60.1-73.4)$ & \\
\hline (2014) & $0 D A$ & USA) from the LIFE study in & Miveasure ti site & Onive or twice & $30-35(n=122)$ & $186.5(99.1-305.1)$ & $62.4(31.9-100.4)$ & $3.2(2.3-4.1)$ & $66.6(60.3-73.2)$ & \\
\hline & & & & & $\geq 35(\mathrm{n}=72)$ & $141.7(58.4-286.5)$ & $60.0(25.5-100.4)$ & $2.8(1.8-3.9)$ & $70.2(61.7-75.9)$ & \\
\hline & & 511 men attending the fertility & & & $18.5-24.99(\mathrm{n}=139)$ & $128.1(21.1-413)^{d}$ & $52.5(7.4-139)^{d}$ & $2.81 .3-5.2) \mathrm{d}$ & $64.0(35.0-80.0)^{\mathrm{d}}$ & \\
\hline $\begin{array}{l}\text { Macdonald et al } \\
\text { (2012) }\end{array}$ & New Zealand & therapeutic procedures at three & $85 \%$, self-reported & once & $25-29.99(\mathrm{n}=253)$ & $135.0(24.1-455)^{\mathrm{d}}$ & $50.0(9.3-152)^{d}$ & $3.0(1.5-5.2) \mathrm{d}$ & $64.5(40.0-81.0)^{\mathrm{d}}$ & \\
\hline & & New Zealand in 2008-2012 & & & $\geq 30(\mathrm{n}=119)$ & $122.1(15.4-407)^{\mathrm{d}}$ & $42.0(7.4-116.5)^{\mathrm{d}}$ & $2.9(1.4-5.2) \mathrm{d}$ & $67.0(44.0-82.0)^{\mathrm{d}}$ & \\
\hline & & & & & $18.5-24.9(\mathrm{n}=123)$ & $257(102-477)$ & $76(35-155)$ & $3.2(2.2-4.2)$ & $49(30-70)$ & \\
\hline Chavarro et al & MA & $\begin{array}{l}483 \text { male partners in subfertile } \\
\text { couples presented for evaluation }\end{array}$ & Measured on site & once & $25-29.9(\mathrm{n}=233)$ & $229(87-414)$ & $81(32-172)$ & $2.9(1.9-4.1)$ & $55(35-69)$ & \\
\hline (2010) & 1012 & $\begin{array}{l}\text { at the MGH Fertility Center in } \\
2000-2006\end{array}$ & 1970 & Fine & $30-34.9(\mathrm{n}=87)$ & $204(92-390)$ & $87(41-154)$ & $3.0(1.8-3.5)$ & $54(30-71)$ & \\
\hline & & & & & $\geq 35(\mathrm{n}=40)$ & $167(78-293)$ & $77(23-148)$ & $2.6(1.9-4.0)$ & $55(25-68)$ & \\
\hline
\end{tabular}

of 435 abstracts. Among these, 24 articles studying the relationship between BMI and sperm parameters seemed potentially appropriate to be included in the meta-analysis (Figure 1).

\section{Description of studies and participants}

The present meta-analysis included 25 studies (Tables 1-2) included in total 26814 participants, among those 2106 participants are from our own study. All these were cross-sectional studies except three prospective studies and one retrospective study that reported crosssectional data as well as a case-control study. Apart from five without mentioned and five self-reported, data of weight and height in other studies were absolutely measured on site by trained personnel. Study participants were recruited from either a general population or the infertility populations from fertility clinic, with one exception of attending physical examination for military service. Sperm analysis was performed followed WHO 1999 guidelines or WHO 2010 guidelines for all studies, except one which is based on the WHO Laboratory Manual for Examination of Human Semen and SpermCervical Mucus Interactions, Cambridge (2001) [19]. Amongst all the studies in this meta-analysis, researched outcomes were as follows: sperm count (19/25), concentration (23/25), volume (20/25), motility (18/25), and progressive motility (12/25). The seven studies [6-9, $20,21]$ with the larger sample size (over 1000) accounted for the major part of the meta-analysis and came to several different results as shown above.

\section{Personal data result}

There was no relationship between BMI and sperm parameters on the whole personal participants 
Table 2: Characteristics of observational studies included in this review (Part 2)

\begin{tabular}{|c|c|c|c|c|c|c|c|c|c|c|}
\hline \multirow[b]{2}{*}{ Study } & \multirow[b]{2}{*}{ Location } & \multirow[b]{2}{*}{ Study group } & \multirow{2}{*}{$\begin{array}{l}\text { Ascertainment } \\
\text { of BMI }\end{array}$} & \multirow{2}{*}{$\begin{array}{l}\text { Repeated } \\
\text { semen } \\
\text { collection }\end{array}$} & \multirow[b]{2}{*}{ BMI distribution } & \multirow{2}{*}{$\begin{array}{l}\text { Total sperm count } \\
\begin{array}{l}\text { Mean } \pm \text { SD } / \text { Median } \\
\text { (IQR) }\end{array}\end{array}$} & \begin{tabular}{|l} 
Sperm \\
concentration
\end{tabular} & \multirow{2}{*}{\begin{tabular}{|l|}
$\begin{array}{l}\text { Semen } \\
\text { volume }\end{array}$ \\
Mean \pm SD/ \\
Median \\
(IQR)
\end{tabular}} & Sperm motility & \begin{tabular}{|l} 
Sperm motility \\
(progressive)
\end{tabular} \\
\hline & & & & & & & $\begin{array}{l}\text { Mean } \pm \quad \text { SD/ } \\
\text { Median (IQR) }\end{array}$ & & $\begin{array}{l}\text { Mean } \pm \text { SD/ } \\
\text { Median (IQR) }\end{array}$ & $\begin{array}{l}\text { Mean } \pm \text { SD/ } \\
\text { Median (IQR) }\end{array}$ \\
\hline \multirow{3}{*}{$\begin{array}{l}\text { Hammiche et al } \\
\text { (2012) }\end{array}$} & \multirow{3}{*}{$\begin{array}{l}\text { The } \\
\text { Netherlands }\end{array}$} & \multirow{3}{*}{$\begin{array}{l}450 \text { men of subfertile couples } \\
\text { visiting a tertiary outpatient } \\
\text { clinic for reproductive } \\
\text { treatment or specialized } \\
\text { medical preconception care in } \\
2007-2010\end{array}$} & \multirow{3}{*}{$\begin{array}{l}\text { Measured on } \\
\text { site }\end{array}$} & \multirow{3}{*}{ once } & $<25(\mathrm{n}=153)$ & $68.6(19.8-183.2)$ & $34.0(8.9-62.3)$ & $3.0(1.9-4.0)$ & & $39.0(22.0-48.5)$ \\
\hline & & & & & $25.0-29.9(\mathrm{n}=225)$ & $49.6(14-124.8)$ & $23.0(6.8-51.5)$ & $2.7(1.5-3.5)$ & & $37.0(21.0-47.0)$ \\
\hline & & & & & $\geq 30(\mathrm{n}=72)$ & $45.9(2.8-147.5)$ & $18.0(1.1-60.3)$ & $2.4(1.6-3.4)$ & & $39.0(23.0-49.0)$ \\
\hline \multirow{4}{*}{$\begin{array}{l}\text { Cheng et al } \\
(2013)\end{array}$} & \multirow{4}{*}{ China } & \multirow{4}{*}{$\begin{array}{l}403 \text { men of subfertile couples } \\
\text { attending the infertility clinic } \\
\text { of Ningxia Medical University } \\
\text { General Hospital in 2008-2012 }\end{array}$} & \multirow{4}{*}{$\begin{array}{l}\text { Measured on } \\
\text { site }\end{array}$} & \multirow{4}{*}{ once } & $18.5-24(\mathrm{n}=182)$ & & $48.36 \pm 28.81$ & $2.52 \pm 0.61$ & $52.73 \pm 22.02$ & $35.82 \pm 15.29$ \\
\hline & & & & & $24-28(\mathrm{n}=154)$ & & $54.09 \pm 32.92$ & $2.55 \pm 0.64$ & $58.71 \pm 20.49$ & $39.53 \pm 14.74$ \\
\hline & & & & & $28-30(\mathrm{n}=35)$ & & $54.32 \pm 31.79$ & $2.62 \pm 0.77$ & $61.23 \pm 21.60$ & $38.47 \pm 16.58$ \\
\hline & & & & & $\geq 30(n=32)$ & & $39.04 \pm 23.02$ & $2.62 \pm 0.78$ & $48.87 \pm 23.18$ & $34.81 \pm 13.73$ \\
\hline \multirow{3}{*}{$\begin{array}{l}\text { Zhu et al } \\
(2014)\end{array}$} & & & & & $18.5-23.9(\mathrm{n}=138)$ & $81.1 \pm 59.3$ & $24.7 \pm 18.6$ & $3.51 \pm 1.4$ & & $26.7 \pm 15.4$ \\
\hline & China & $\begin{array}{l}318 \text { infertile men attending the } \\
\text { infertility clinic in } 2012\end{array}$ & $\begin{array}{l}\text { Measured } \\
\text { site }\end{array}$ & once & $24-27.9(\mathrm{n}=116)$ & $56.2 \pm 49.7$ & $18.4 \pm 16.3$ & $3.22 \pm 1.43$ & & $24.3 \pm 14.9$ \\
\hline & & & & & $\geq 28(n=64)$ & $45.4 \pm 41.1$ & $14.8 \pm 12.6$ & $3.27 \pm 1.87$ & & $20.2 \pm 14.0$ \\
\hline & & 245 normozoospermic male & & & $20.1-25(\mathrm{n}=96)$ & & $39 \pm 14$ & & & \\
\hline Koloszar et al & Hungary & patients of reproductive age & Measured & NA & $25.1-30(\mathrm{n}=91)$ & & $37 \pm 14$ & & & \\
\hline & & problems & & & $>30(n=58)$ & & $29 \pm 12$ & & & \\
\hline & & 260 male partners of pregnant & & & $<25(\mathrm{n}=127)$ & $316.9(275.7-364.6)^{b}$ & $80.5(69.4-93.3)^{b}$ & $3.9(3.7-4.2) \mathrm{b}$ & $50.2(48.0-52.4)^{\mathrm{b}}$ & \\
\hline Aleksejev et al & Estonia & women presenting for prenatal & $\begin{array}{l}\text { Measured } \\
\text { site }\end{array}$ & NA & $25.0-29.9(\mathrm{n}=95)$ & $260.6(218.0-311.6)^{b}$ & $66.1(56.7-77.0)^{\mathrm{b}}$ & $3.9(3.6-4.3) \mathrm{b}$ & $52.0(49.6-54.5)^{\mathrm{b}}$ & \\
\hline & & & & & $\geq 30(\mathrm{n}=38)$ & $223.6(164.2-304.5)^{\mathrm{b}}$ & $69.4(52.7-91.4)^{\mathrm{b}}$ & $3.2(2.8-3.8) \mathrm{b}$ & $54.2(50.8-57.5)^{\mathrm{b}}$ & \\
\hline & & 177 infertile men aged 23- & & & $<24(\mathrm{n}=58)$ & $146.39 \pm 111.87$ & $53.09 \pm 34.30$ & $2.71 \pm 0.92$ & $58.50 \pm 24.19$ & $50.60 \pm 23.23$ \\
\hline Bai et al (2014) & China & 50 from infertility clinic of & $\begin{array}{l}\text { Measured } \\
\text { site }\end{array}$ & once & $24-27.99(\mathrm{n}=60)$ & $131.14 \pm 94.90$ & $57.88 \pm 37.57$ & $2.27 \pm 0.98$ & $58.00 \pm 23.96$ & $51.70 \pm 21.92$ \\
\hline & & Kunming Medical University & & & $>28(\mathrm{n}=59)$ & $103.75 \pm 78.38$ & $52.44 \pm 34.06$ & $1.96 \pm 0.7^{\mathrm{e}}$ & $54.17 \pm 26.06$ & $46.49 \pm 25.18$ \\
\hline & & 168 patients attendeding & & & $20-24.9(\mathrm{n}=34)$ & & $37.5(5.4-119)^{\mathrm{c}}$ & $2.8(0.9-6)^{\mathrm{e}}$ & $50(0-85)^{\mathrm{e}}$ & \\
\hline Mormandi et al & Argentina & $\begin{array}{l}\text { the andrology section of the } \\
\text { endocrinology of Hospital }\end{array}$ & Measured & at least once & $25-29.9(\mathrm{n}=100)$ & & $30(3.6-325)^{\mathrm{c}}$ & $2.5(0.2-7)^{\mathrm{c}}$ & $60.5(0-90)^{\mathrm{e}}$ & \\
\hline & & $\begin{array}{l}\text { Durand for infertility in 2008- } \\
2010\end{array}$ & site & & $\geq 30(\mathrm{n}=34)$ & & $33.5(2-268)^{\mathrm{C}}$ & $2.3(0.3-5.2)^{\mathrm{C}}$ & $47.5(10-85)^{\mathrm{c}}$ & \\
\hline & & 166 men aged 18 years & & & $18.5-24.9(\mathrm{n}=45)$ & $205(7-1862)^{\mathrm{e}}$ & $53(1.3-222)^{\mathrm{c}}$ & & $63(17-74)^{\mathrm{c}}$ & \\
\hline Andersen et al & Norwa & and older from two & Measured & once & $25-29.9(\mathrm{n}=52)$ & $190(7-601)^{\mathrm{e}}$ & $60(3.6-350)^{\mathrm{c}}$ & & $41(1-76)^{\mathrm{e}}$ & \\
\hline (2015) & & advertisement and a fertility & site & once & $30-34.9(n=31)$ & $244(6-1290)^{\mathrm{e}}$ & $54.9(3.8-305)^{\mathrm{c}}$ & & $43(10-70)^{\mathrm{e}}$ & \\
\hline & & clinic in 2008-2013 & & & $\geq 35(\mathrm{n}=38)$ & $121(20-1127)^{c}$ & $41.5(3.0-281)^{\mathrm{c}}$ & & $30(0-43)^{\mathrm{e}}$ & \\
\hline & & 151 male patients living as a & & & $20.1-25.0(\mathrm{n}=66)$ & $115.84 \pm 65.1$ & & & $47.56 \pm 18.2$ & \\
\hline Hajshafiha et al & Iran & $\begin{array}{l}\text { partner in an infertile couple } \\
\text { fertile }\end{array}$ & $\begin{array}{l}\text { Measured } \\
\text { site }\end{array}$ & twice & $25.1-30.0(\mathrm{n}=66)$ & $116.3 \pm 71.6$ & & & $41.78 \pm 19.6$ & \\
\hline & & seeking infertility treatment & & & $>30.0(\mathrm{n}=19)$ & $115.36 \pm 74.8$ & & & $46.52 \pm 18.7$ & \\
\hline & & 153 men from couples & & & $<24.9(\mathrm{n}=74)$ & & $61.0 \pm 45.8$ & $3.8 \pm 1.6$ & $54.4 \pm 11.1$ & \\
\hline Rybar et al & Czech & attending an infertility clinic & NA & NA & $25-29.9(\mathrm{n}=63)$ & & $60.5 \pm 39.5$ & $3.6 \pm 1.6$ & $53.4 \pm 9.8$ & \\
\hline & & $\begin{array}{l}\text { or more to achieve pregnancy } \\
\text { without success }\end{array}$ & & & $>30(\mathrm{n}=16)$ & & $70.8 \pm 43.6$ & $4.5 \pm 1.8$ & $53.8 \pm 12.0$ & \\
\hline & & 150 general-based populations & & & $19.0-24.9(\mathrm{n}=50)$ & $211.1 \pm 30.2^{\mathrm{a}}$ & $66.0 \pm 5.3 \mathrm{a}$ & $3.2 \pm 0.6^{\mathrm{a}}$ & & $48.4 \pm 4.4 \mathrm{a}$ \\
\hline $\begin{array}{l}\text { Vignera et al } \\
(2012)\end{array}$ & Italy & $\begin{array}{l}\text { containing } 50 \text { normal-weight, } \\
50 \text { overweight and } 50 \text { obese }\end{array}$ & $\begin{array}{l}\text { Measured } \\
\text { site }\end{array}$ & twice & $25.1-29.9(\mathrm{n}=50)$ & $225.1 \pm 44.4^{a}$ & $68.2 \pm 11.0 \mathrm{a}$ & $3.3 \pm 0.4^{\mathrm{a}}$ & & $20.2 \pm 4.0 \mathrm{a}$ \\
\hline & & men selected randomly & & & $30.1-44.0(\mathrm{n}=50)$ & $191.7 \pm 26.4^{\mathrm{a}}$ & $57.9 \pm 9.7 \mathrm{a}$ & $3.3 \pm 0.8^{\mathrm{a}}$ & & $23.2 \pm 6.0 \mathrm{a}$ \\
\hline & & & & & $18.5-24.9(\mathrm{n}=36)$ & $156.42 \pm 27.96^{\mathrm{a} a}$ & $51.30 \pm 8.48^{\mathrm{a}}$ & $3.36 \pm 0.35^{\mathrm{a}}$ & $43.75 \pm 5.07^{\mathrm{a}}$ & \\
\hline Gutorova et al & Russia & born in Arkhangelsk or lived & Measured & once & $25.1-29.9(\mathrm{n}=44)$ & $215.50 \pm 22.95^{a}$ & $67.94 \pm 6.94 \mathrm{a}$ & $3.24 \pm 0.28^{\mathrm{a}}$ & $52.10 \pm 4.16^{a}$ & \\
\hline & & & & & $\geq 30.1(n=19)$ & $113.35 \pm 34.64^{\mathrm{a}}$ & $40.20 \pm 10.48^{a}$ & $2.88 \pm 0.42^{\mathrm{a}}$ & $37.23 \pm 6.28^{\mathrm{a}}$ & \\
\hline & & 26 infertile men aged $21-50$ & & & $18.5-24(\mathrm{n}=26)$ & & $59.58 \pm 30.30$ & $2.46 \pm 0.94$ & $66.38 \pm 14.30$ & $57.54 \pm 16.94$ \\
\hline Bai et al (2015) & China & $\begin{array}{l}\text { from infertility clinic and } 26 \\
\text { healthy control from maternity }\end{array}$ & $\begin{array}{l}\text { Measured } \\
\text { site }\end{array}$ & once & $24-28(\mathrm{n}=12)$ & & $62.58 \pm 29.30$ & $2.51 \pm 0.74$ & $62 \pm 14.52$ & $54.67 \pm 15.74$ \\
\hline & & clinic for the second child & & & $\geq 28(\mathrm{n}=14)$ & & $67.50 \pm 36.76$ & $2.53 \pm 1.03$ & $61.57 \pm 13.58$ & $53.64 \pm 13.78$ \\
\hline
\end{tabular}

${ }^{\mathrm{a}}$ Mean \pm SE; ${ }^{\mathrm{b}}$ mean $(95 \% \mathrm{CI}) ;{ }^{\mathrm{c}}$ mean $(\mathrm{IQR}) ;{ }^{\mathrm{d}}$ median (10th-90th percentile); ${ }^{\mathrm{e}}$ median (range)

(Supplementary Table 1). Compared with normal weight, weight abnormalities had no effect on the low sperm parameters of total people (Supplementary Table 2), and had no effect on the low sperm parameters of fertile parts only (Supplementary Table 3), while obesity was associated with significantly increased ORs for low sperm count $(\mathrm{OR}=2.69)$, sperm concentration $(\mathrm{OR}=1.87)$, sperm motility $(\mathrm{OR}=2.47)$ and sperm progressive motility $(\mathrm{OR}=2.03)$ in infertile men (Supplementary Table 4) (all results were adjusted for participants age).

\section{Impact of BMI on sperm parameters}

In this review, we used a total of 24 studies and personal data to perform the meta-analysis. With the normal weight participants as the reference group, the standardized weighted mean differences (SMD) of sperm parameters of abnormal weight group was calculated (Table 3, Figure 2 for data of total sperm count). From the meta-analysis, we found overweight decreased the quality of total sperm count and semen volume $(P=0.000$ and 0.002$)$, obesity decreased the quality of total sperm count, sperm concentration, and semen volume $(P=0.001$, 


\section{A Authors}

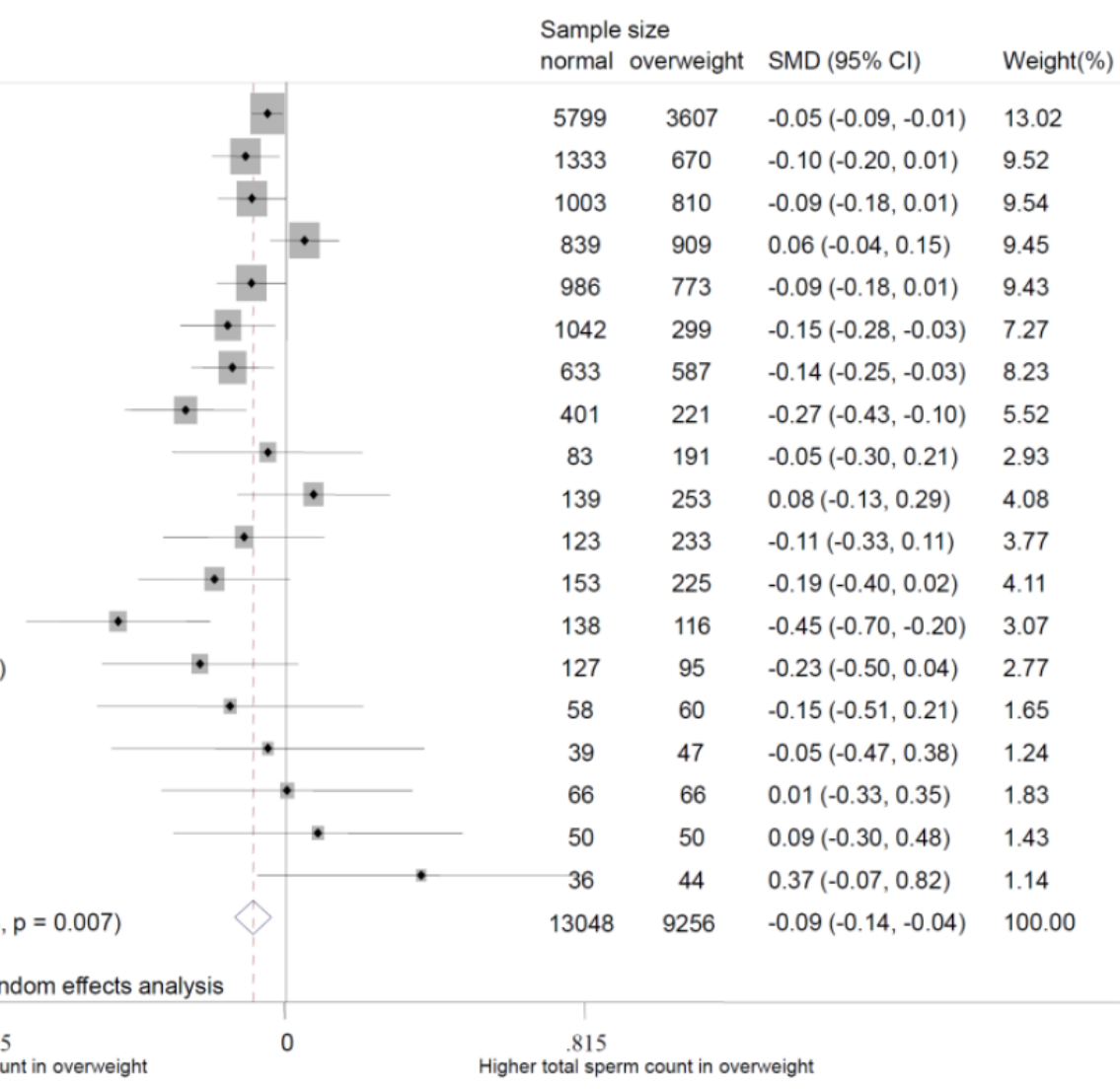

Belloc et al. (2014)

Guo et al.(unpublished)

Paasch et al (2010)

Shayeb et al (2011)

Aggerholm et al (2008)

Jensen et al (2004)

Duits et al (2010)

Xiao et al (2013)

Eisenberg et al (2014)

Macdonald et al (2013)

Chavarro et al (2010)

Hammiche et al (2012)

Zhu et al (2014)

Ehala-Aleksejev et al (2015)

Bai et al (2014)

Andersen et al (2015)

Hajshafiha et al (2013)

Vignera et al (2012)

Gutorova et al (2014)

Overall (I-squared $=50.2 \%, p=0.007$ )

sperm count in overweight

Lower total sperm count in overweight

\begin{tabular}{llll}
\multicolumn{2}{l}{ Sample size } & & \\
Normal & Obese & SMD $(95 \% \mathrm{Cl})$ & Weight $(\%)$ \\
5799 & 764 & $-0.19(-0.27,-0.12)$ & 9.22 \\
1333 & 103 & $-0.09(-0.29,0.11)$ & 6.71 \\
1003 & 245 & $-0.09(-0.23,0.05)$ & 8.02 \\
839 & 269 & $0.11(-0.03,0.25)$ & 8.07
\end{tabular}

\section{B Authors}

Belloc et al (2014)

Guo et al (unpublished)

Paasch et al (2010)

Shayeb et al (2011)

Aggerholm et al (2008)

Duits et al (2010)

Xiao et al (2013)

Eisenberg et al (2014)

Macdonald et al (2013)

Chavarro et al (2010)

Hammiche et al (2012)

Zhu et al (2014)

Ehala-Aleksejev et al (2015)

Bai et al (2014)

Andersen et al (2015)

Hajshafiha et al (2013)

Vignera et al (2012)

Gutorova et al (2014)

Overall (I-squared $=68.5 \%, p=0.000$ )

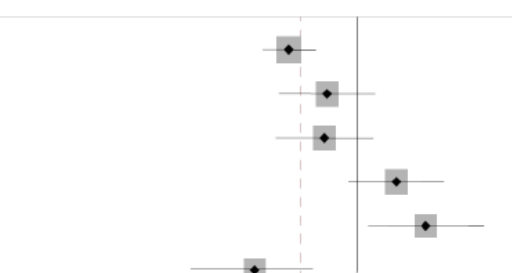

$986 \quad 163 \quad 0.20(0.03,0.36) \quad 7.45$

$633 \quad 146 \quad-0.29(-0.47,-0.11) \quad 7.14$

$401 \quad 164-0.28(-0.46,-0.10) \quad 7.10$

$83 \quad 194 \quad-0.18(-0.43,0.08) \quad 5.56$

$139 \quad 119 \quad-0.07(-0.32,0.17) \quad 5.80$

$123 \quad 127 \quad-0.27(-0.52,-0.02) \quad 5.72$

$153 \quad 72 \quad-0.19(-0.48,0.09) \quad 5.14$

$138 \quad 64 \quad-0.66(-0.96,-0.36) \quad 4.76$

$127 \quad 38 \quad-0.38(-0.74,-0.01) \quad 3.87$

$58 \quad 59 \quad-0.44(-0.81,-0.08) \quad 3.84$

$39 \quad 64 \quad-0.07(-0.46,0.33) \quad 3.47$

$66 \quad 19 \quad-0.01(-0.52,0.50) \quad 2.45$

$50 \quad 50 \quad-0.10(-0.49,0.30) \quad 3.54$

$36 \quad 19 \quad-0.27(-0.82,0.29) \quad 2.14$

NOTE: Weights are from random effects analysis

$120062679 \quad-0.16(-0.25,-0.07) \quad 100.00$

$$
-.962
$$

0

Lower total sperm count in obese

962

Higher total sperm count in obese

Figure 2: Forest plot of abnormal body mass and total sperm count compared with normal body mass. Each point represents a separate study for the indicated association. A. overweight; B. obese. 
Table 3: SMD and 95\% CI of sperm parameters in abnormal weight groups

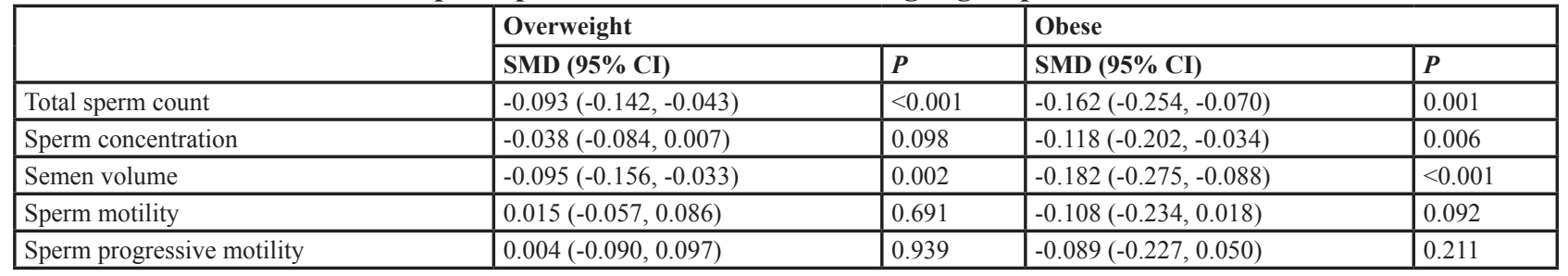

All SMD are relative to normal weight (BMI between 18.5-25.0).

0.006 and 0.000 , respectively), while changes of sperm motility didn't show significant statistical difference, and are not shown on a specific figure. For dose-response analysis, only seven articles had the mean values of the BMI categories that were used in our analysis. The results showed sperm count, sperm concentration and semen volume were weakened with the increase of BMI with the $\mathrm{P}$ values were $0.000,0.038$ and 0.003 , respectively (Figure $3)$. And, for every 5 -unit increase in BMI, the SMD fell $2.4 \%, 1.3 \%$ and $2.0 \%$ compared with normal weight, respectively.

\section{Sensitivity analyses}

To reduce the heterogeneity between these studies, random effected models were performed in this meta analysis. Then, we conducted the sensitive analyses to access whether modification of the inclusion criteria affected the final results. The results showed that the SMD of progressive motility in obese group changed when eliminated data from Aggerholm et al (2008) [9], while the outcome of other groups was not qualitatively changed with or without any study. Also, there was no obvious influence on the results after taking out of the data from our data or Belloc et al (2014) [8] (Figure 4).

\section{Subgroup analyses}

Subgroup analyses were conducted based on the ethnicity of participants. The results showed that the sperm parameters were different in Caucasian and Asian population in some BMI groups, such as total sperm count,
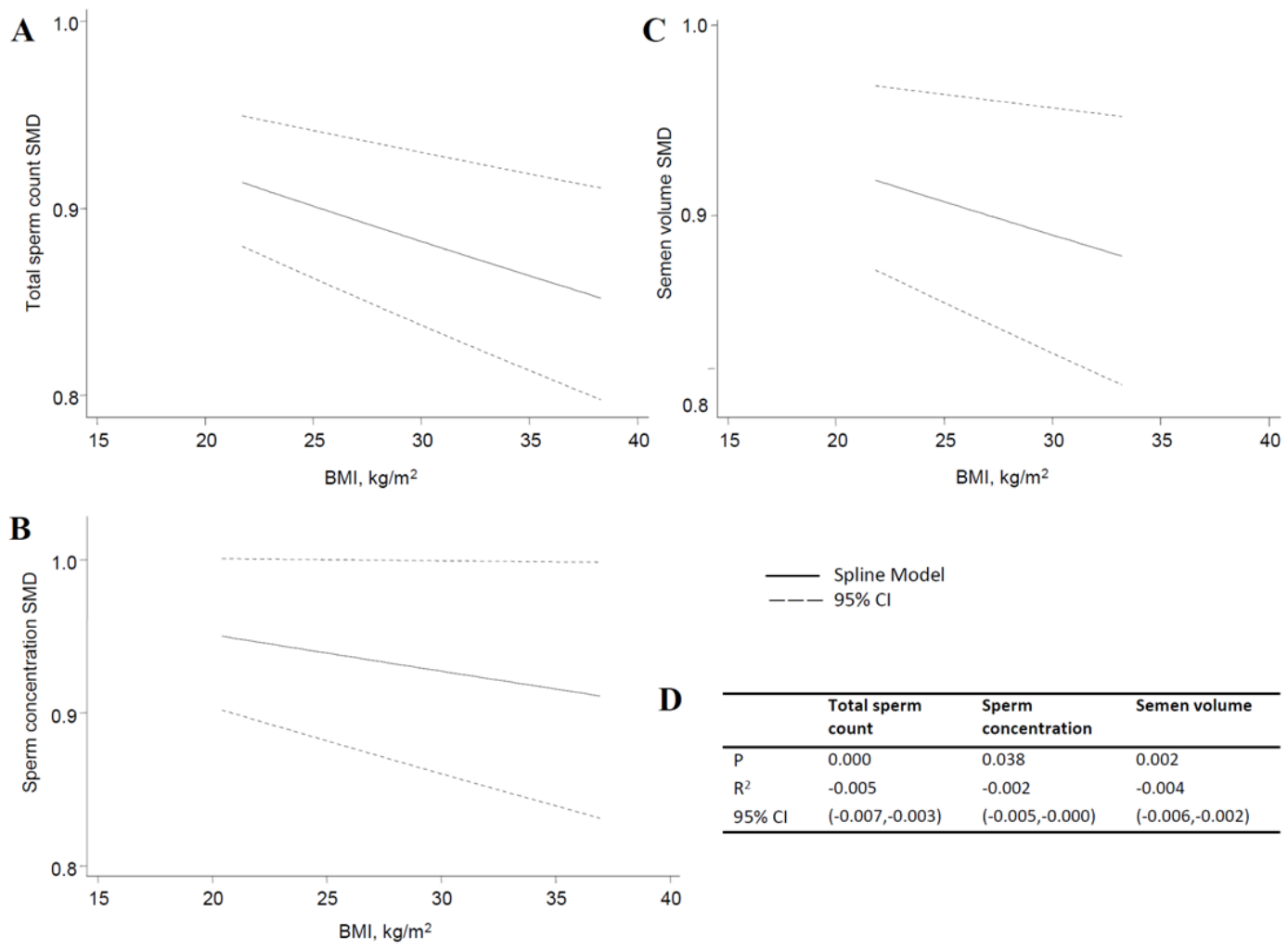

_ Spline Model $--95 \% \mathrm{Cl}$

D

\begin{tabular}{llll}
\hline & $\begin{array}{l}\text { Total sperm } \\
\text { count }\end{array}$ & $\begin{array}{l}\text { Sperm } \\
\text { concentration }\end{array}$ & Semen volume \\
\hline $\mathrm{P}$ & 0.000 & 0.038 & 0.002 \\
$\mathrm{R}^{2}$ & -0.005 & -0.002 & -0.004 \\
$95 \% \mathrm{Cl}$ & $(-0.007,-0.003)$ & $(-0.005,-0.000)$ & $(-0.006,-0.002)$ \\
\hline
\end{tabular}

Figure 3: Dose-response of total sperm count, sperm concentration and semen volume. A. total sperm count; B. sperm concentration; C. semen volume; D. the parameters of dose-response. 
semen volume and sperm motility in the obese group, but the effect of BMI was similar (Figure 5).

\section{Assessment of publication bias}

Using Egger's test and Begg's funnel plot to evaluate the publication bias of studies, the results provided no evidence in each sperm parameter of different groups (Figure 6).

\section{Trial sequential analyses}

We used trial sequential analyses to calculate the required information size of different sperm parameters
A

B

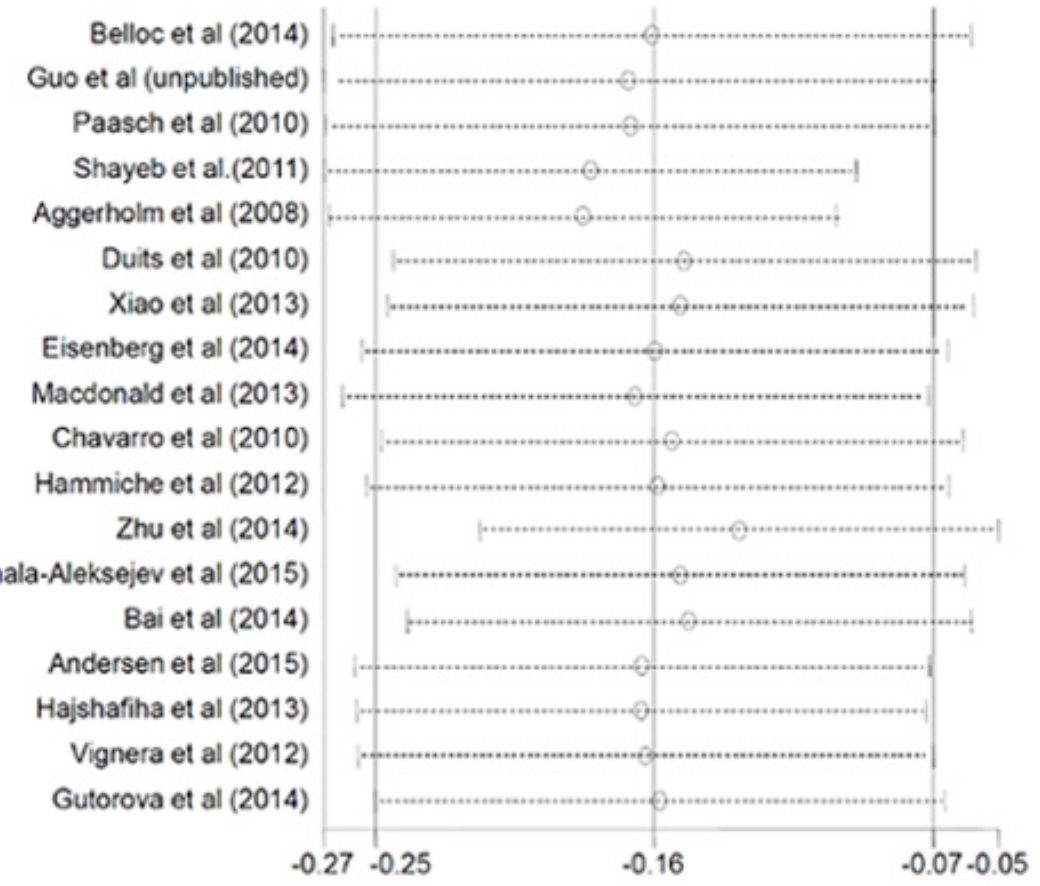

Gutorova et al (2014)
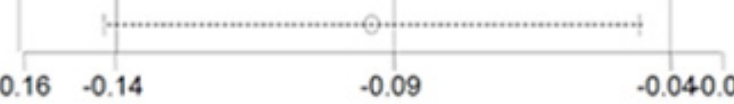

$-.10052662(-.15818164,-.04287162)$

$-.09258314(-.14680965,-.03835664)$

$-.09426779(-.14878501,-.03975057)$

$-.10689684(-.15473928,-.0590544)$

$-09408832(-.14849825,-.03967838)$

$-.08824411(-.14004856,-.03643966)$

$-.08883256(-.14126456,-.03640057)$

$-.08158311(-.12919429,-.03397192)$

-09464119 (-.14575338, -.04352899)

$-.09990542(-.14987965,-.04993119)$

$-.09252422(-.14390135,-.04114708)$

$-.08869065(-.13930681,-.0380745)$

$-.0801461(-.12374752,-.03654467)$

$-.08895629(-.13903847,-.03887411)$

$-09217072(-.14273725,-.04160417)$

$-.09374338(-.1442771,-.04320966)$

$.09499871(-.14559597,-.04440145)$

$-.09566073(-.14578335,-.04553811)$

$.09693177(-.1445318,-.04933175)$

$-.09283948(-.14225867,-.0434203)$

$-.16311677(-.26934299,-.05689055)$

$-.16839902(-.26657802,-.07022)$

$-.17010751(-.2709344,-.0692806)$

$-.18333031(-.2709569,-.09570374)$

$-.18603651(-.26938882,-.10268418)$

$-.15261087(-.2491982,-.05602353)$

$-.15384787(-.25082392,-.05687182)$

$-.16213773(-.25904128,-.06523418)$

$-.16848031(-.26546359,-.07149702)$

$-.15639414(-.25267696,-.06011133)$

$-.16114876(-.25758424,-.06471325)$

$-.13450144(-.22044413,-.04855875)$

$-.15346768(-.24756648,-.05936889)$

$-.15069531(-.24394329,-.05744733)$

$-.16617967(-.26125452,-.07110484)$

$-.16647972(-.26074576,-.07221369)$

$-.16513902(-.26033673,-.06994132)$

$-.16025434(-.25436994,-.06613875)$

$-.16214918(-.25434145,-.06995692)$

Figure 4: Sensitivity analysis about total sperm count. A. overweight; B. obese. 
Table 4: Statistically significant changed metabolites in seminal plasma

\begin{tabular}{|l|l|l|l|}
\hline Metabolite & $\boldsymbol{P}$ Value & Median case & Median control \\
\hline Sorbitol & 0.004809 & 1.56884 & 0.875969 \\
\hline 2-Hydroxyglutaric acid & 0.005751 & 0.177469 & 0.097649 \\
\hline Fumaric acid & 0.010213 & 0.187117 & 0.241223 \\
\hline Maltose & 0.014895 & 241000 & 94518 \\
\hline Fructose & 0.014956 & 58.0786 & 41.5207 \\
\hline Arabionse & 0.019451 & 0.056726 & 0.025847 \\
\hline Spermine & 0.020477 & 0.017984 & 0.003356 \\
\hline 3a,7a,12a-Trihydroxy-5a-cholestanoic acid & 0.023849 & 0.008571 & 0.010245 \\
\hline Spermidine & 0.023851 & 0.260766 & 0.156707 \\
\hline Palmitic acid & 0.025077 & 0.464602 & 0.375263 \\
\hline Ribose & 0.026356 & 1.18142 & 0.800664 \\
\hline Cholesterol & 0.030544 & 0.743243 & 0.412022 \\
\hline Dehydroascorbic Acid & 0.030742 & 0.053261 & 0.00141 \\
\hline Creatinine & 0.035289 & 15.5022 & 19.9561 \\
\hline Stearic acid & 0.046684 & 0.217045 & 0.153005 \\
\hline Aminomalonic acid & 0.048856 & 1.22205 & 1.98582 \\
\hline
\end{tabular}

in different groups. The results showed that none of the numbers included in the meta-analysis exceed the required information size except semen volume of obese group and some boundary TSA ignoring due to too little information use (Figure 7). Among the positive results in metaanalysis, TSA showed that the cumulative Z-curve (blue line) did cross both the conventional boundary $(P=0.05)$ and the trial sequential monitoring boundary in total sperm count and semen volume of overweight group and obese group, sperm concentration of obese group, indicating the positive results were confirmed. In contrast, the negative results of TSA of other sperm groups turned out to be true positive.

\section{Metabolite identification of seminal plasma}

The seminal plasma of four groups depending on $\mathrm{BMI}$ and sperm parameters (NN: both normal; NA: normal BMI and abnormal sperm parameters; AN: abnormal BMI (obese) and normal sperm parameters; AA: abnormal BMI (obese) and abnormal sperm parameters) were used to perform the metabolomic analysis. (Sperm parameters normal means total sperm count, sperm concentration, semen volume, motility are all above WHO reference lower limits. In contrast, it will be defined as abnormal). We got the preliminary significant changed metabolites profile compared the results of group AA with NN. After eliminated the different ones between group NN and NA, as well as some changed ones between group NN and AN, we caught the final different metabolites list (Table 4), and analyzed metabolic pathways using MetaboAnalyst (http:// www.metaboanalyst.ca/). The results showed 5 metabolic pathways were significantly changed, among which the three lowest $P$ values were of arginine and proline metabolism, beta-alanine metabolism and glutathione metabolism (Table 5). Interestingly, the mutual metabolites of these pathways that showed different concentrations of group AA compared with NN were spermidine and spermine. The concentrations of spermidine and spermine were significantly higher in group AA than group NN.

\section{DISCUSSION}

In the meta-analysis, we found that high BMI decreased sperm quality such as sperm count, concentration, and semen volume rather than sperm motility (overall or progressive). Meanwhile the trial sequential analyses confirmed the relationship to some extent according to the calculation of the required information size.

Our result for the association between BMI and sperm quality is consistent with some large studies [6, $8,20]$, though they showed influence on different sperm parameters. In contrast, our calculation got a different conclusion to the previous relevant meta-analysis published in 2010, which found no relationship between BMI and sperm quality in generally $[10,11]$. But it is incredible Sermondade et al found abnormal body weight elevated the risk of oligozoospermia or azoospermia though the whole analysis had a negative result in another meta-analysis published in 2013 [11]. The results of our own unpublished data showed obese men were more like to have total sperm count, sperm concentration and sperm motility below the WHO lower reference limits than normal weight men in infertile part. All of these convince us that there exists a relationship between body weight and sperm quality in general population more than the special crowed. The sample size may be the main issue and the current meta-analysis recruited 25 studies including 26814 individuals, which made up for the deficiency of 
Caucasian

Belloc et al (2014)

Paasch et al (2010)

Shayeb et al (2011)

Aggerholm et al (2008)

Jensen et al (2004)

Duits et al (2010)

Andersen et al (2015)

Vignera et al (2012)

Gutorova et al (2014)

Subtotal $(\mathrm{I}$-squared $=46.6 \%, p=0.059$ )

$-0.05(-0.09,-0.01) \quad 13.02$

$-0.09(-0.18,0.01) \quad 9.54$

$0.06(-0.04,0.15) \quad 9.45$

$-0.09(-0.18,0.01) \quad 9.43$

$-0.15(-0.28,-0.03) \quad 7.27$

$-0.14(-0.25,-0.03) \quad 8.23$

$-0.05(-0.47,0.38) \quad 1.24$

$0.09(-0.30,0.48) \quad 1.43$

$0.37(-0.07,0.82) \quad 1.14$

Asian

Guo et al (unpublished)

Xiao et al (2013)

Zhu et al (2014)

Bai et al (2014)

Subtotal (I-squared $=63.5 \%, p=0.041$ )

Mixed

Eisenberg et al (2014)

Macdonald et al (2013)

Chavarro et al (2010)

Hammiche et al (2012)

Ehala-Aleksejev et al (2015)

Hajshafiha et al (2013)

Subtotal (I-squared $=0.0 \%, p=0.420$ )

Overall (I-squared $=50.2 \%, p=0.007$ )

$-0.06(-0.11,-0.01) \quad 60.75$

NOTE: Weights are from random effects analysis

$$
-.815
$$

Lower total sperm count in overweight

$-0.10(-0.20,-0.01) \quad 9.52$

$-0.27(-0.43,-0.10) \quad 5.52$

$-0.45(-0.70,-0.20) \quad 3.07$

$-0.15(-0.51,0.21) \quad 1.65$

$-0.23(-0.39,-0.07) \quad 19.76$

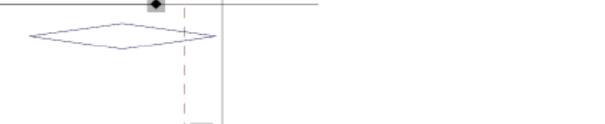

Authors

$\operatorname{SMD}(95 \% \mathrm{Cl})$

Weight(\%)

\section{Caucasian}

Belloc et al (2014)

Paasch et al (2010)

Shayeb et al (2011)

Aggerholm et al (2008)

Duits et al (2010)

Andersen et al (2015)

Vignera et al (2012)

Gutorova et al (2014)

Subtotal $($ I-squared $=78.2 \%, p=0.000)$

Asian

Guo et al (unpublished)

Xiao et al (2013)

Zhu et al (2014)

Bai et al (2014)

Subtotal $($ I-squared $=70.3 \%, p=0.018$ )

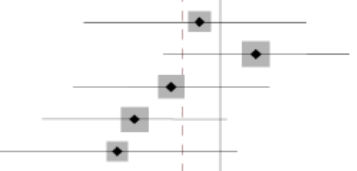

$-0.05(-0.30,0.21) \quad 2.93$

$0.08(-0.13,0.29) \quad 4.08$

$-0.11(-0.33,0.11) \quad 3.77$

$-0.19(-0.40,0.02) \quad 4.11$

$-0.23(-0.50,0.04) \quad 2.77$

$0.01(-0.33,0.35) \quad 1.83$

$-0.08(-0.18,0.01) \quad 19.49$

$-0.09(-0.14,-0.04) \quad 100.00$

815

Higher total sperm count in overweight

\section{Mixed}

Eisenberg et al (2014)

Macdonald et al (2013)

Chavarro et al (2010)

Hammiche et al (2012)

Ehala-Aleksejev et al (2015)

Hajshafiha et al (2013)

Subtotal ( -squared $=0.0 \%, p=0.738$ )

Overall $(\mathrm{I}$-squared $=68.5 \%, \mathrm{p}=0.000)$

$-0.19(-0.27,-0.12) \quad 9.22$

$-0.09(-0.23,0.05) \quad 8.02$

$0.11(-0.03,0.25) \quad 8.07$

$0.20(0.03,0.36) \quad 7.45$

$-0.29(-0.47,-0.11) \quad 7.14$

$-0.07(-0.46,0.33) \quad 3.47$

$-0.10(-0.49,0.30) \quad 3.54$

$-0.27(-0.82,0.29) \quad 2.14$

$-0.07(-0.21,0.07) \quad 49.03$

NOTE: Weights are from random effects analysis

$-.962$

$-0.09(-0.29,0.11) \quad 6.71$

$-0.28(-0.46,-0.10) \quad 7.10$

$-0.66(-0.96,-0.36) \quad 4.76$

$-0.44(-0.81,-0.08) \quad 3.84$

$-0.34(-0.57,-0.11) \quad 22.41$

$-0.18(-0.43,0.08) \quad 5.56$

$-0.07(-0.32,0.17) \quad 5.80$

$-0.27(-0.52,-0.02) \quad 5.72$

$-0.19(-0.48,0.09) \quad 5.14$

$-0.38(-0.74,-0.01) \quad 3.87$

$-0.01(-0.52,0.50) \quad 2.45$

$-0.19(-0.31,-0.07) \quad 28.55$

$-0.16(-0.25,-0.07) \quad 100.00$

Lower total sperm count in obese

0

Figure 5: Subgroup analysis based on ethnicity about total sperm count. A. overweight; B. obese. 
A Begg's funnel plot with pseudo $95 \%$ confidence limits

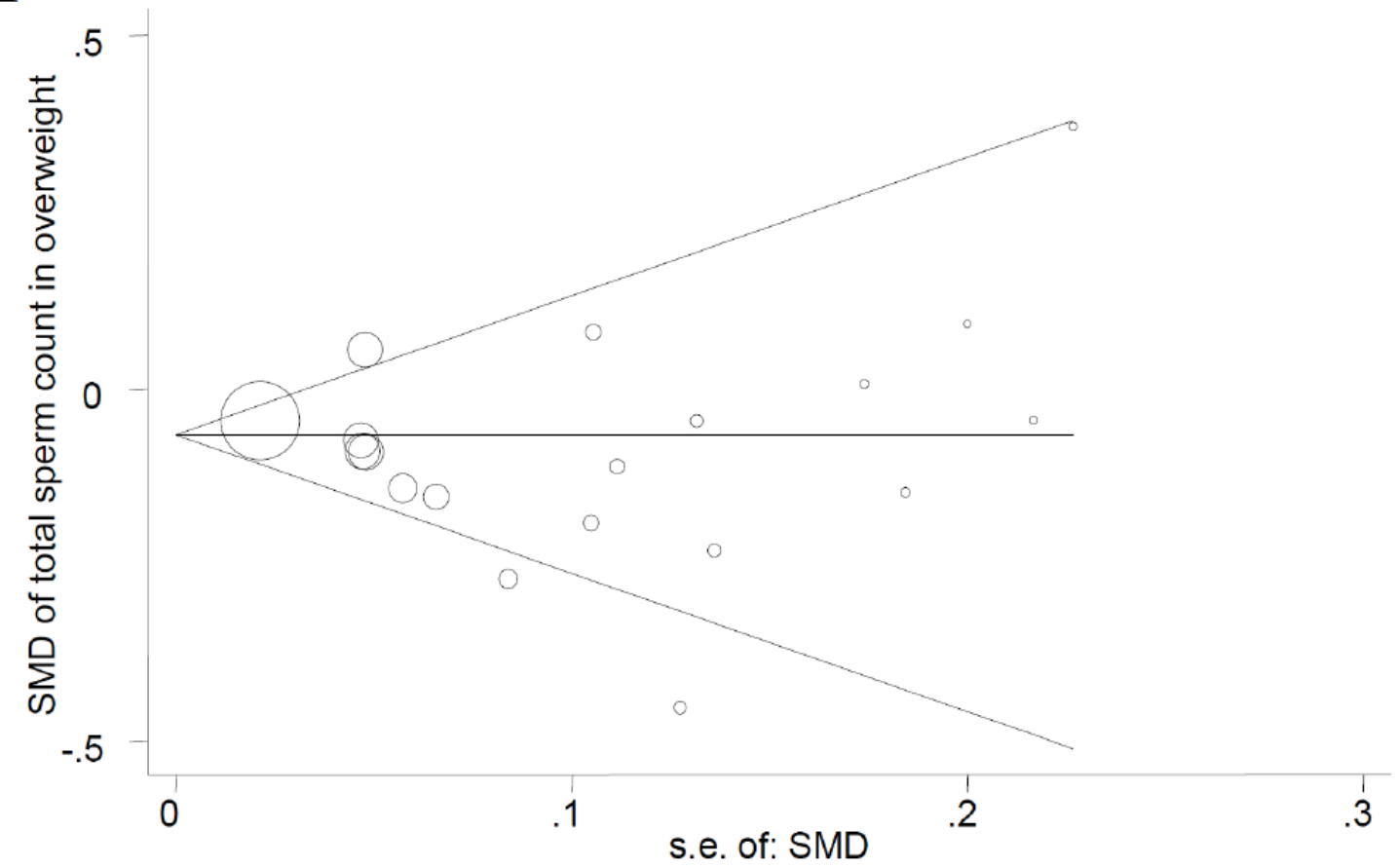

Begg's Test: $P=0.624 \quad$ Egger's Test: $P=0.150$

B Begg's funnel plot with pseudo $95 \%$ confidence limits

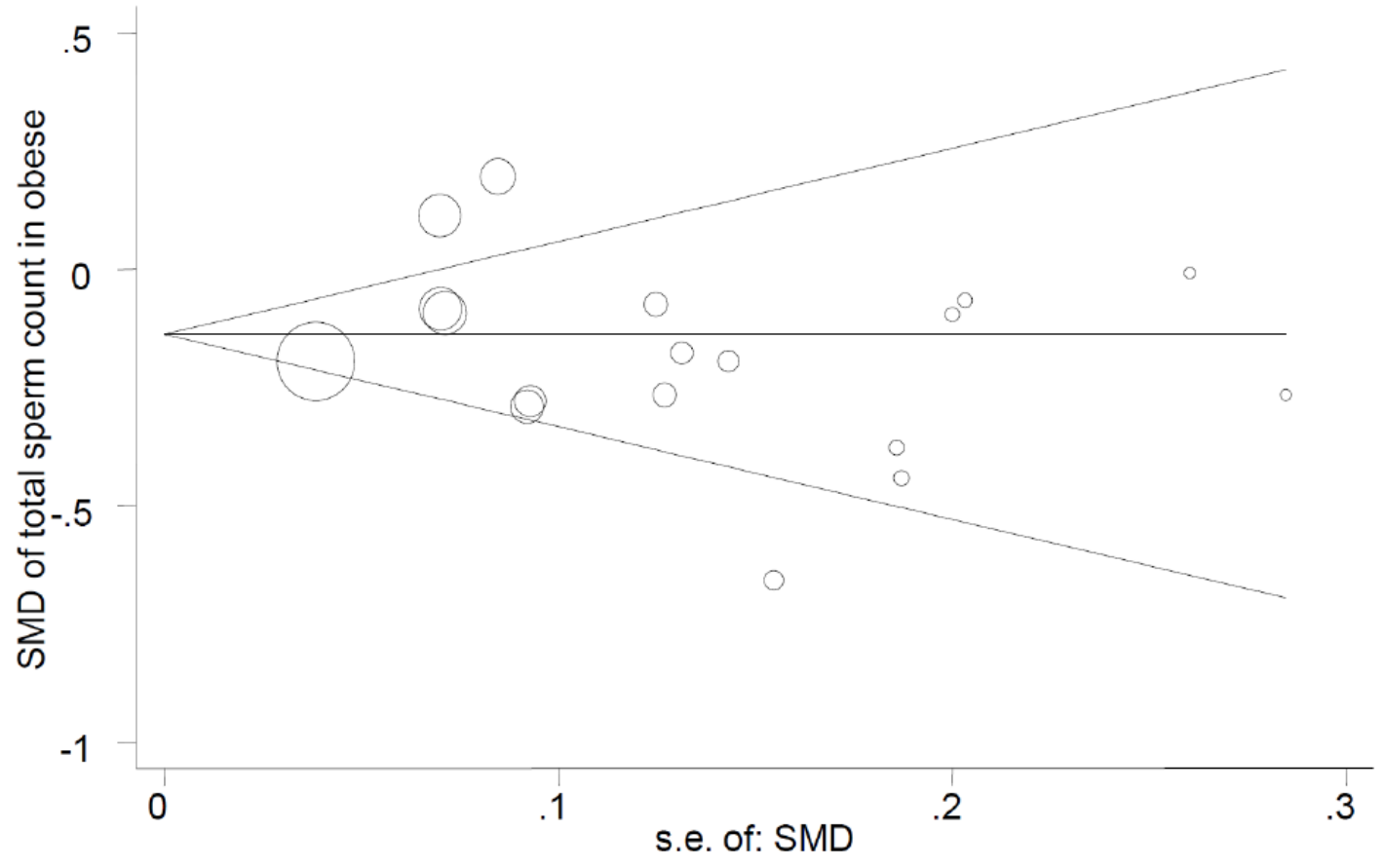

Begg's Test: $P=0.705 \quad$ Egger's Test: $P=0.274$

Figure 6: Funnel plot of observational studies about total sperm count. A. overweight; B. obese. 
the previous meta-analysis published in 2013 containing 13077 participants and our own data unpublished including 2106 individuals. In addition, the trial sequence analyses increased the credibility of the results avoiding the false positive in the meta-analysis.

The effect of obesity on the traditional sperm parameters is thought to be multifactorial and the proposed pathophysiological mechanisms underlying these sophisticated relationships have been raised from endocrinology to psychology. First of all, probably of more concern is the changes of hypothalamic-pituitarygonadal (HPG) axis. Aromatization activity increased by the elevated white adipose tissue of obese men converts testosterone to estrogens, and the enhancive leptin coming from the same source decreases the production of testosterone from Leydig cells [22]. Besides, hypoxemia caused from sleep apnea of obese men is another reason of a decline in morning testosterone concentrations [23]. Decreased testosterone and increased estrogen disrupt the negative feedback loop of the HPG axis, which
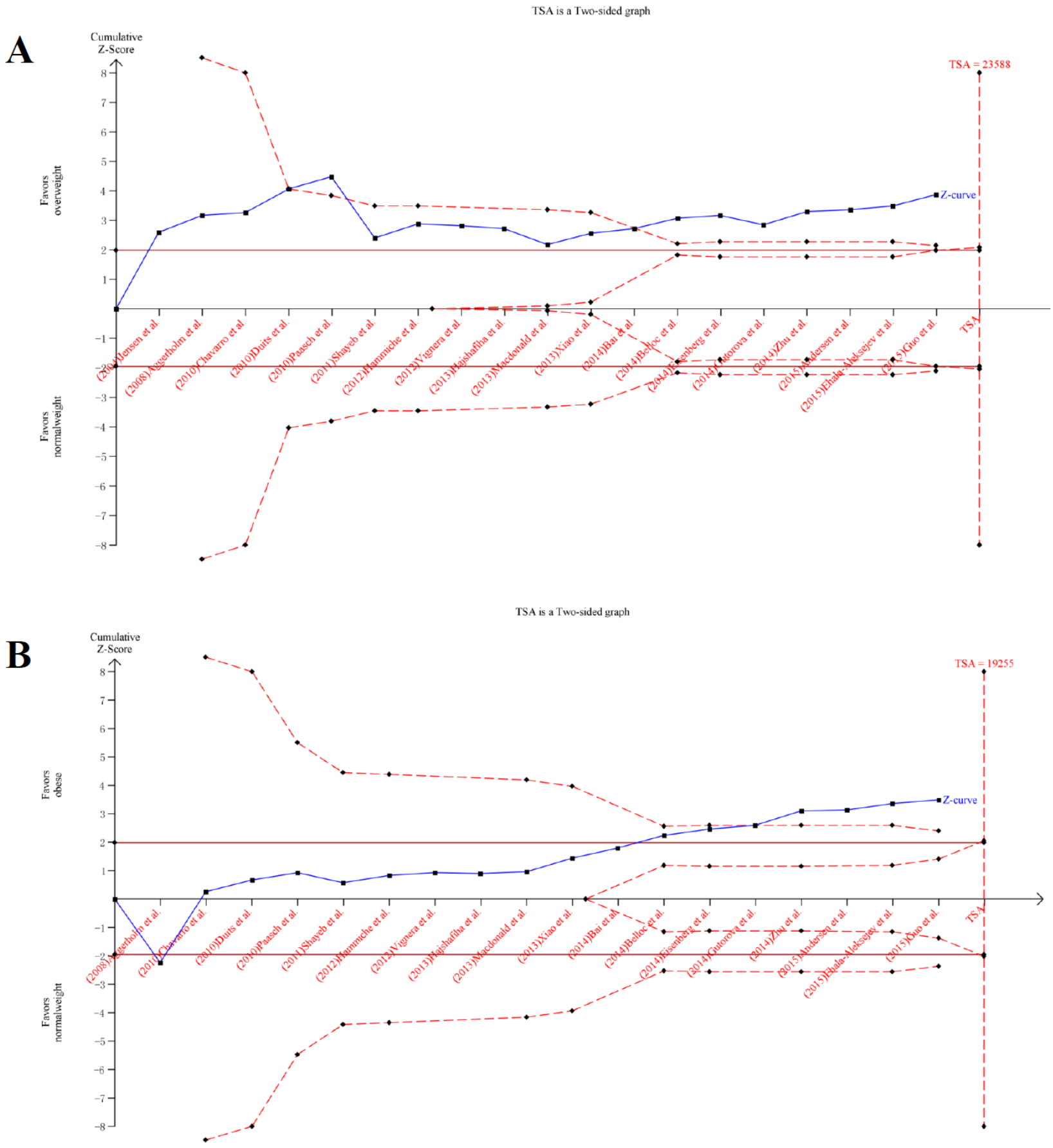

Figure 7: Trial sequential analysis about total sperm count. A. overweight; B. obese. 
Table 5: Pathway enrichment of significant changed metabolites

\begin{tabular}{|l|l|}
\hline Pathway name & $\boldsymbol{P}$ value \\
\hline Arginine and proline metabolism & 0.000395 \\
\hline beta-Alanine metabolism & 0.008016 \\
\hline Glutathione metabolism & 0.014501 \\
\hline Fatty acid biosynthesis & 0.023529 \\
\hline Starch and sucrose metabolism & 0.024442 \\
\hline Citrate cycle (TCA cycle) & 0.095485 \\
\hline Alanine, aspartate and glutamate metabolism & 0.11355 \\
\hline Fatty acid elongation in mitochondria & 0.12688 \\
\hline Pentose phosphate pathway & 0.14869 \\
\hline Butanoate metabolism & 0.18254 \\
\hline Galactose metabolism & 0.18669 \\
\hline Nicotinate and nicotinamide metabolism & 0.19901 \\
\hline Phenylalanine metabolism & 0.20307 \\
\hline Fructose and mannose metabolism & 0.21516 \\
\hline Fatty acid metabolism & 0.22313 \\
\hline Tyrosine metabolism & 0.32016 \\
\hline Amino sugar and nucleotide sugar metabolism & 0.36109 \\
\hline
\end{tabular}

interfere with the normal progress of spermatogenesis. Secondly, obese men have an increased level of oxidative stress resulting from an increase in seminal macrophage activation, which damage sperm DNA integrity [24]. Thirdly, some researchers proposed another hypothesis that the thermal effect resulting from increased scrotal adiposity could harm sperm cells [25]. Finally, obesity can also be related to erectile dysfunction, and to sexuality in a reverse fashion, due to the psychological impact [26]. Because obesity can be thought as a metabolic disease, we expect new finding from the metabonomics that no one has ever been involved in. For the metabolomic analysis, we found spermidine and spermine had higher concentrations in the group of obese men with abnormal sperm quality. Spermidine and spermine are polyamines, and are essential to male reproductive processes such as testicular development and spermatogenesis. Although evidence for the occurrence of polyamines indicates that it is beneficial and indispensable to spermatogenesis, an excess is detrimental to the progress. Halmekytö M et al studied the effect of superabundant polyamines on spermatogenesis by the transgenic mice [27]. They found the first and second generation male offspring displayed reduced reproductive performance, even infertile or no spermatogenesis. Which implies us it might be the polyamines such as spermidine and spermine that play an important role in the poor sperm quality resulted from obesity. Of course, the precise mechanism needs more relevant deeper researches.

There are several limitations to our study. First, we didn't obtain the original data through contacting the corresponding authors. All the information in the metaanalysis relied on published articles only. It is inevitable that the unity and credibility of data is disturbed to a certain extent because of the variety of BMI categories, statistical approach and outcomes. We used the SMD and converted data format by the accepted formula to reduce the influence as far as possible. Besides, due to the limitation of data sources, the use of different boundaries for normal, overweight and obese in Chinese studies was different from others, which may affect the final results. However, because people of different ethnicities experience various extrinsic factors and they have different genetic sources, the physique is different between Asians and Western populations and we have conducted subgroup analyses based on the ethnicity of participants and different classification standards to reflect the impact of such difference brought about. Second, considering the data processing and statistical aggregation, some relevant articles accessing effect of BMI on sperm quality were excluded even though they maybe provide useful information. In order to verify the reliability of the results, we performed the trial sequence analyses, which confirmed the validity of the present meta-analysis though the sample size didn't meet the requirement. Third, BMI was considered as the unique surrogate of body fat content to evaluate the influence of obesity on sperm quality, and other information such as the relationship between waist circumference $[16,18]$, or waist-to-hip [18] ratio, or waist-to-height [18] and male infertility was ignored. BMI is flawed and not a perfect index because it has been questioned about its thresholds for overweight and obesity [28], and it does not distinguish fat from fat-free mass [29]. In spite of it, compared with other indicators, BMI is still the most widely used and relatively convenient, which suggesting our findings be more universal and more suitable for the application.

Our results have confirmed that obesity is a 
pernicious factor of sperm quality. While we look forward to springing out large sample researches to further validate about the relevance between obesity and sperm motility and morphology. According to the conclusion, it would be natural to assume that weight loss will have a beneficial effect on fertility of obese men. However, the truth is still unknown. Several relevant articles have been published about weight loss through dietary, exercise interventions, or bariatric surgery and male fertility. Most reports paid more attention to the effect on the reproductive hormones rather than sperm parameters and the results were ambiguous. Niskanen et al [30] and Kaukua et al [31] found weight loss increased testosterone level, while Leenen et al [32] showed there was no correlation between weight loss and total testosterone level. Likewise, Hakonsen et al [33] found weight loss could improve total sperm count, while Sermondade et al [34] got the opposite conclusion. Further study assessing the effect of weight loss (especially some amusing ways such as changes in meal timing [35]) on the improvement of sperm quality or not is warranted. Besides, the finding of metabolomic analysis suggests us considering from the point of small molecules to explain the phenomenon and apply to clinical therapy. Of course, it also needs more in-depth researches to explore the mechanism and utilize rationally.

Based on this study, sperm quality decreases along with BMI increasing, suggesting obesity may be a detrimental factor of male infertility, though lacking of the raw data may influence the accuracy of the results. Further research is required to identify the role of obesity in male sterility.

\section{MATERIALS AND METHODS}

\section{Search strategy and selection criteria}

We searched relevant studies published until June 2015 about BMI and sperm parameters from PubMed, Embase, Web of Science, and Wanfang database without language restriction. The following combined text and MeSH terms were used: ('overweight' OR 'weight' OR 'obesity' OR 'BMI' OR 'body fat' OR 'body weight' OR 'body mass index' OR 'adiposity' OR 'IBW' OR 'ideal weight') AND ('sperm' OR 'semen' OR 'spermatozoa' OR 'sperm count' OR 'sperm concentration' OR 'semen quality' OR 'semen parameters' OR 'sperm quantity' OR 'total sperm count' OR 'oligozoospermia' OR 'azoospermia' OR 'semen volume' OR 'sperm motility' OR 'spermatids' OR 'spermatocytes' OR 'spermatogonia'). We also searched the references of key articles to identify other relevant studies.

After a primary screen of all titles retrieved from the database, we excluded reviews, studies without BMI or sperm data or studies without results on the relationship between BMI and sperm parameters, experimental or interventional studies, mechanism articles, female researches, studies restricted to men with a particular pathology (such as a varicocele) and studies comparing exposed/non-exposed men. Then the full texts of potentially eligible articles were retrieved, regardless of population size, origin, age or ethnicity. Two review authors independently examined these articles and extracted the data. Any disagreements were resolved by discussion between them. Data retrieved included study characteristics and sperm parameters.

\section{Recruitment and screening of personal participants}

We recruited 1711 and 653 participants from infertility clinic and maternity clinic respectively to collect semen samples by masturbation and got their basic information (age, weight, height) by self-report. Semen analysis was carried out according to the WHO laboratory manual (2010). After eliminated participants with 1. known azoospermia; 2 . other reproductive diseases such as varicocele; 3. BMI $<18.5$; 4. data incomplete, 2106 individuals were involved in the analysis finally (including 1461 infertile men and 645 fertile men). We computed the relationship between BMI and sperm parameters on the whole personal participants by regression analysis. Then, we calculated the odds risk (OR) under WHO reference lower limits ( 5 th percentiles) (sperm count $<39$ million, concentration $<15 \mathrm{million} / \mathrm{ml}$, volume $<1.5 \mathrm{ml}$, motility $<40 \%$ and progressive motility $<32 \%$ ) in different BMI groups of total participants, fertile men and infertile men respectively.

\section{Data synthesis and analysis}

Meta-analysis was performed using studies that including one or more sperm parameter in the BMI categories as following: mean or median total sperm count, sperm concentration, semen volume, sperm motility, or sperm progressive motility. Data were converted to the form of mean and standard deviation in order to facilitate the calculation. BMI was divided into three levers by WHO criteria: 18.5-24.9 (normal weight), 25.0-29.9 (overweight) and above 30 (obese) $\mathrm{kg} / \mathrm{m}^{2}$, except in some Chinese studies that defining 24.0, and 28.0 as boundaries. Participants with a BMI between 18.5-24.9 or 18.523.9 were deemed as the reference group. Each sperm parameter of each abnormal weight group was compared to the reference group using SMD, which were calculated by mean, standard deviation and sample size using Stata version 12.0. Then, we performed the dose-response analysis about normal weight, overweight and obese with the data reported in these studies [36]. The values of sperm parameters for normal weight, overweight, obese and the mean BMI of the categories had to be available. We calculated a $P$ value for linearity to test the hypothesis that the coefficient was different from 0 . 
The $\mathrm{I}^{2}$ statistic was performed to assess possible heterogeneity between studies. We used the random-effect model to evaluate SMD if the $P$ value for heterogeneity was $\leq 0.10$ or $\mathrm{I}^{2} \geq 50 \%$ that demonstrating a high extent of heterogeneity between studies [37]. Furthermore, a sensitive analysis was conducted to evaluate the effect of a single study on the whole effect. Subgroup analysis was performed to assess the effects between each subgroup based on ethnicity of study population at the same time. We utilized Egger's test and Begg's funnel plot to perform a diagnosis of publication bias. Trial sequential analysis (TSA) was used to minimize the risk of type-I errors of meta-analysis [38]. The required information size was evaluated according to a type-I errors of 5\%, a power of $80 \%$ and the pooled estimate of all included trials as anticipated variance. After adjusted data of targetmetabolites by quality control samples, the Wilcoxon ranksum test was used for two-group tests of metabolomic analysis of our own study. Stata version 12.0 and TSA software (The Copenhagen Trial Unit, Center for Clinical Intervention Research, Denmark) was used to perform all statistical, and $P<0.05$ was defined as statistically significant.

\section{Metabolomic analysis of seminal plasma}

The seminal plasma of infertile participants of our own participants were used to perform the metabolomic analysis according to the previous study [39]. Briefly, seminal plasma was mixed with methionine sulphone (MetSul) and D-camphor-10-sulfonic acid (CSA) that used as internal standards. After removing proteins by filtrating, the mixtures were used to perform CE-TOFMS analysis. Sample was injected with a pressure injection of $50 \mathrm{mbar}$ for $10 \mathrm{~s}$ and $25 \mathrm{~s}$ for anion and cation mode, respectively. The participants were divided into four groups: NN $(n=$ $55)$, NA $(n=52)$, AN $(n=12)$, and AA $(n=9)$, while others were kicked out $(n=1333)$.

\section{Authors' contributions}

Conceived and designed the experiments: WW DG QT. Searched for and selected the publications: DG WW QT. Analyzed the data: DG WW YC MT SQ. Contributed reagents/materials/analysis tools: WW MC CL HD YX LH DC JS XW. Wrote and revised the paper: DG WW QT XW.

\section{ACKNOWLEDGMENTS}

This work was supported by National Natural Science Foundation of China (No. 81302457, 81673217, 81401213), Jiangsu Natural Science Foundation (No. BK20130894), China Postdoctoral Science Foundation (No. 2015T80571), University Natural Science Research
Project in Jiangsu Province (No. 13KJB330002), and the Priority Academic Program for the Development of Jiangsu Higher Education Institutions (Public Health and Preventive Medicine).

\section{CONFLICTS OF INTEREST}

The authors declare no conflict of interest.

\section{REFERENCE}

1. Gurunath S, Pandian Z, Anderson RA, Bhattacharya S. Defining infertility - a systematic review of prevalence studies. Hum Reprod Update. 2011; 17:575-88.

2. Mascarenhas MN, Flaxman SR, Boerma $T$, Vanderpoel $\mathrm{S}$, Stevens GA. National, regional, and global trends in infertility prevalence since 1990: a systematic analysis of 277 health surveys. PLoS Med. 2012; 9:e1001356.

3. Hafekost K, Lawrence D, Mitrou F, O'Sullivan TA, Zubrick SR. Tackling overweight and obesity: does the public health message match the science? BMC Med. 2013; 11:41.

4. Xu X, Zhou L, Miao R, Chen W, Zhou Y, Pang Q, Qu K, Liu C. Association of cancer mortality with postdiagnosis overweight and obesity using body mass index. Oncotarget. 2016; 7:5023-29. doi: 10.18632/oncotarget.6517.

5. Ng M, Fleming $\mathrm{T}$, Robinson M, Thomson B, Graetz N, Margono C, Mullany EC, Biryukov S, Abbafati C, Abera SF, Abraham JP, Abu-Rmeileh NM, Achoki T, et al. Global, regional, and national prevalence of overweight and obesity in children and adults during 1980-2013: a systematic analysis for the Global Burden of Disease Study 2013. Lancet. 2014; 384:766-81.

6. Shayeb AG, Harrild K, Mathers E, Bhattacharya S. An exploration of the association between male body mass index and semen quality. Reprod Biomed Online. 2011; 23:717-23.

7. Duits FH, van Wely M, van der Veen F, Gianotten J. Healthy overweight male partners of subfertile couples should not worry about their semen quality. Fertil Steril. 2010; 94:1356-59.

8. Belloc S, Cohen-Bacrie M, Amar E, Izard V, Benkhalifa M, Dalléac A, de Mouzon J. High body mass index has a deleterious effect on semen parameters except morphology: results from a large cohort study. Fertil Steril. 2014; 102:1268-73.

9. Aggerholm AS, Thulstrup AM, Toft G, Ramlau-Hansen $\mathrm{CH}$, Bonde JP. Is overweight a risk factor for reduced semen quality and altered serum sex hormone profile? Fertil Steril. 2008; 90:619-26.

10. MacDonald AA, Herbison GP, Showell M, Farquhar CM. The impact of body mass index on semen parameters and reproductive hormones in human males: a systematic review with meta-analysis. Hum Reprod Update. 2010; 16:293-311. 
11. Sermondade N, Faure C, Fezeu L, Shayeb AG, Bonde JP, Jensen TK, Van Wely M, Cao J, Martini AC, Eskandar M, Chavarro JE, Koloszar S, Twigt JM, et al. BMI in relation to sperm count: an updated systematic review and collaborative meta-analysis. Hum Reprod Update. 2013; 19:221-31.

12. Hajshafiha M, Ghareaghaji R, Salemi S, Sadegh-Asadi N, Sadeghi-Bazargani H. Association of body mass index with some fertility markers among male partners of infertile couples. Int J Gen Med. 2013; 6:447-51.

13. Macdonald AA, Stewart AW, Farquhar CM. Body mass index in relation to semen quality and reproductive hormones in New Zealand men: a cross-sectional study in fertility clinics. Hum Reprod. 2013; 28:3178-87.

14. Mormandi EA, Otero P, Bertone AL, Calvo M, Astarita G, Kogovsek N, Levalle O. [Body weight increase and quality of semen: a controversial association]. [Article in Spanish]. Endocrinol Nutr. 2013; 60:303-07.

15. Ehala-Aleksejev K, Punab M. The different surrogate measures of adiposity in relation to semen quality and serum reproductive hormone levels among Estonian fertile men. Andrology. 2015; 3:225-34.

16. Eisenberg ML, Kim S, Chen Z, Sundaram R, Schisterman EF, Buck Louis GM. The relationship between male BMI and waist circumference on semen quality: data from the LIFE study. Hum Reprod. 2014; 29:193-200.

17. Leisegang K, Bouic PJ, Menkveld R, Henkel RR. Obesity is associated with increased seminal insulin and leptin alongside reduced fertility parameters in a controlled male cohort. Reprod Biol Endocrinol. 2014; 12:34.

18. Tsao CW, Liu CY, Chou YC, Cha TL, Chen SC, Hsu CY. Exploration of the association between obesity and semen quality in a 7630 male population. PLoS One. 2015; 10:e0119458.

19. Gutorova NV, Kleshchyov MA, Tipisova EV, Osadchuk LV. Effects of overweight and obesity on the spermogram values and levels of reproductive hormones in the male population of the European north of Russia. Bull Exp Biol Med. 2014; 157:95-98.

20. Paasch U, Grunewald S, Kratzsch J, Glander HJ. Obesity and age affect male fertility potential. Fertil Steril. 2010; 94:2898-901.

21. Jensen TK, Andersson AM, Jørgensen N, Andersen AG, Carlsen E, Petersen JH, Skakkebaek NE. Body mass index in relation to semen quality and reproductive hormones among 1,558 Danish men. Fertil Steril. 2004; 82:863-70.

22. Hausman GJ, Barb CR, Lents CA. Leptin and reproductive function. Biochimie. 2012; 94:2075-81.

23. Caprio M, Isidori AM, Carta AR, Moretti C, Dufau ML, Fabbri A. Expression of functional leptin receptors in rodent Leydig cells. Endocrinology. 1999; 140:4939-47.

24. Zorn B, Vidmar G, Meden-Vrtovec H. Seminal reactive oxygen species as predictors of fertilization, embryo quality and pregnancy rates after conventional in vitro fertilization and intracytoplasmic sperm injection. Int J Androl. 2003; 26:279-85.

25. Shafik A, Olfat S. Lipectomy in the treatment of scrotal lipomatosis. Br J Urol. 1981; 53:55-61.

26. Esposito K, Giugliano F, Di Palo C, Giugliano G, Marfella R, D'Andrea F, D'Armiento M, Giugliano D. Effect of lifestyle changes on erectile dysfunction in obese men: a randomized controlled trial. JAMA. 2004; 291:2978-84.

27. Halmekytö M, Hyttinen JM, Sinervirta R, Utriainen M, Myöhänen S, Voipio HM, Wahlfors J, Syrjänen S, Syrjänen K, Alhonen L, Jänne J. Transgenic mice aberrantly expressing human ornithine decarboxylase gene. J Biol Chem. 1991; 266:19746-51.

28. Prentice AM, Jebb SA. Beyond body mass index. Obes Rev. 2001; 2:141-47.

29. Mullie P, Vansant G, Hulens M, Clarys P, Degrave E. Evaluation of body fat estimated from body mass index and impedance in Belgian male military candidates: comparing two methods for estimating body composition. Mil Med. 2008; 173:266-70.

30. Niskanen L, Laaksonen DE, Punnonen K, Mustajoki P, Kaukua J, Rissanen A. Changes in sex hormone-binding globulin and testosterone during weight loss and weight maintenance in abdominally obese men with the metabolic syndrome. Diabetes Obes Metab. 2004; 6:208-15.

31. Kaukua J, Pekkarinen T, Sane T, Mustajoki P. Sex hormones and sexual function in obese men losing weight. Obes Res. 2003; 11:689-94.

32. Leenen R, van der Kooy K, Seidell JC, Deurenberg P, Koppeschaar HP. Visceral fat accumulation in relation to sex hormones in obese men and women undergoing weight loss therapy. J Clin Endocrinol Metab. 1994; 78:1515-20.

33. Håkonsen LB, Thulstrup AM, Aggerholm AS, Olsen J, Bonde JP, Andersen CY, Bungum M, Ernst EH, Hansen ML, Ernst EH, Ramlau-Hansen CH. Does weight loss improve semen quality and reproductive hormones? Results from a cohort of severely obese men. Reprod Health. 2011; $8: 24$.

34. Sermondade N, Massin N, Boitrelle F, Pfeffer J, Eustache F, Sifer C, Czernichow S, Lévy R. Sperm parameters and male fertility after bariatric surgery: three case series. Reprod Biomed Online. 2012; 24:206-10.

35. Pivovarova O, Gögebakan Ö, Sucher S, Groth J, Murahovschi V, Kessler K, Osterhoff M, Rudovich N, Kramer A, Pfeiffer AF. Regulation of the clock gene expression in human adipose tissue by weight loss. Int $\mathrm{J}$ Obes (Lond). 2016; 40:899-906.

36. Rong Y, Chen L, Zhu T, Song Y, Yu M, Shan Z, Sands A, Hu FB, Liu L. Egg consumption and risk of coronary heart disease and stroke: dose-response meta-analysis of prospective cohort studies. BMJ. 2013; 346:e8539.

37. Higgins JP, Thompson SG. Quantifying heterogeneity in a meta-analysis. Stat Med. 2002; 21:1539-58.

38. Møller CH, Penninga L, Wetterslev J, Steinbrüchel DA, 
Gluud C. Clinical outcomes in randomized trials of off- vs. on-pump coronary artery bypass surgery: systematic review with meta-analyses and trial sequential analyses. Eur Heart J. 2008; 29:2601-16.
39. Chen M, Zhou K, Chen X, Qiao S, Hu Y, Xu B, Xu B, Han X, Tang R, Mao Z, Dong C, Wu D, Wang Y, et al. Metabolomic analysis reveals metabolic changes caused by bisphenol A in rats. Toxicol Sci. 2014; 138:256-67. 